\title{
Long term multi-wave length observations campaign of IGR J17091-3624 and IGR J17098-3628, two transient black hole candidates discovered by INTEGRAL: latest results.
}

\author{
Fiamma Capitanio * \\ INAF -IASF Roma, Italy \\ E-mail: fiamma.capitaniodiasf-roma.inaf.it \\ Marcello Giroletti \\ INAF-IRA Bologna, Italy \\ Giovanni De Cesare, Antonella Tarana \\ INAF -IASF Roma, Italy
}

Angela Bazzano, Pietro Ubertini

INAF -IASF Roma, Italy

Paolo Persi, Anna Rosa Marenzi

INAF -IASF Roma, Italy

\begin{abstract}
IGR J17091-3624 and IGR J17098-3628 are two X-ray transients discovered by INTEGRAL and classified as black hole candidates (BHCs). The behaviour of these two sources has been monitored thanks to the analysis of a multi-wavelength data sets collected by different instruments from 2005 until 2008. Since part of this work will be published in the Astrophysical Journal (January 2009), this proceeding is mostly dedicated to the latest outcoming results of this study developed during 2008. In particular we show here the results of the 2008 Swift ToO data analysis during which IGR J17098-3628 was in a bright soft state while IGR J17091-3624 was not detectable. We also report in this paper on a tentative searching of the counterpart of IGR J17091-3624 in the infrared archives.
\end{abstract}

7th INTEGRAL Workshop

September 8-11 2008

Copenhagen, Denmark

${ }^{*}$ This work has been supported by the Italian Space Agency through grants I/008/07/0 and I/088/06/0. We acknowledge the use of public data from the Swift data archive and all the Swift team for its support. 


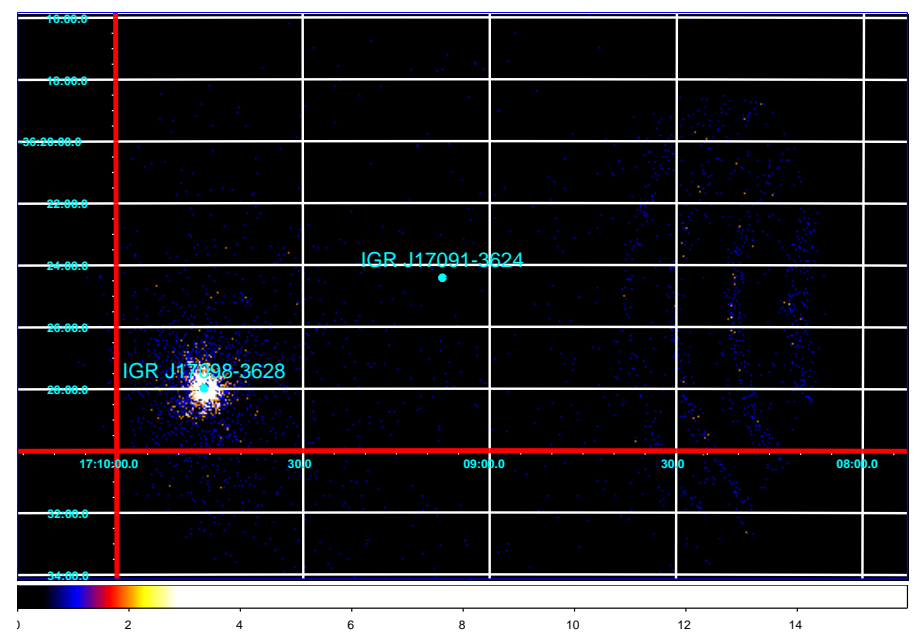

Figure 1: Swift/XRT 2-10 keV image of IGR J17098-3628 observed in June 2008.

\section{Introduction}

IGR J17098-3628 has been regularly detected by INTEGRAL and RXTE over the entire period of the observational campaign starting from its discover until nowdays; it was also observed with pointed observations by XMM and Swift/XRT in 2005 and 2006 and exhibited flux variations not linked with the change of any particular spectral features.

IGR J17091-3624 was instead initially in quiescence (after a period of activity between 2003 April and 2004 April) and it was then detected again in outburst in the XRT field of view during a Swift observation of IGR J17098-3628 on 2007 July 9. The observations during quiescence, performed by Swift and XMM provided an upper limit to the $0.2-10 \mathrm{keV}$ luminosity: $7 \times 10^{32} \mathrm{ergs}$ $\mathrm{s}^{-1}$ [1], while the observations in outburst covered the transition from the hard to the soft state [1]. Moreover, Kennea \& Capitanio (2007) obtained a refined X-ray position for IGR J17091-3624 from the Swift/XRT observations during the outburst in 2007, the new position is inconsistent with the previously proposed radio counterpart. A new compact radio source consistent with the new X-ray position was identify by Capitanio et al. (2009).

\section{The 2008 Swift ToO}

The angular distance (9.1') of the two sources does not allows RXTE/ASM to propely associate flux variations to one of the two sources. This issue was solved with Swift ToOs pointed towards the two sources zone. The observation on July 2007 pointed out that the increasing flux of RXTE/ASM was due mainly to the IGR J17091-3624 outburst while IGR J17098-3628 was almost constant. On the contrary the June 2008 ToO revealed IGR J17098-3628 at 50 mCrab, while IGR J17091-3624 was not detected up to $3 \sigma$ level. Figure 1 shows the two sources region image (2-10 keV) of the XRT June 2008 ToO.

The 2008 ToO consisted of three pointings: the first and the shortest one, was performed in imaging mode in order to verify the status of the two sources: IGR J17091-3624 was not detectable 


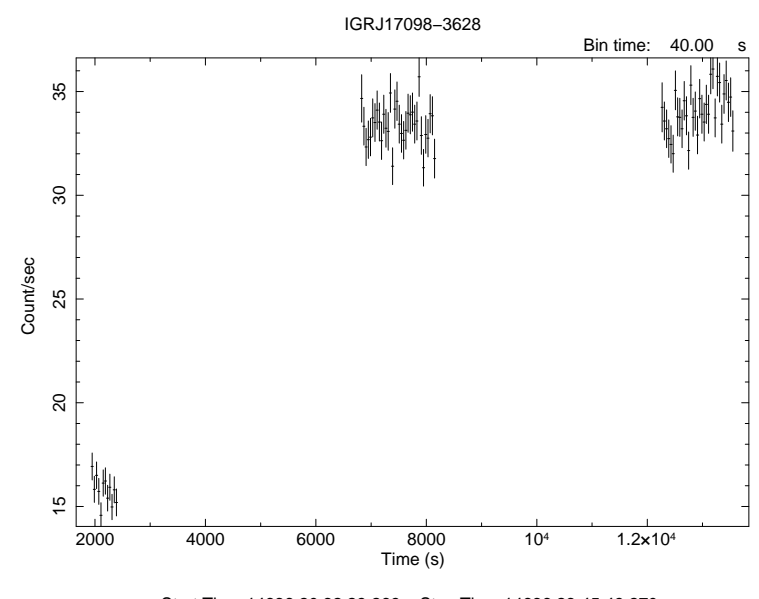

Figure 2: Swift/XRT 2-10 keV light curve of IGR J17098-3628 during the 200819 June pointing.

$\begin{array}{lccccc}\text { Date } & \mathrm{n}_{H} & \mathrm{~T}_{\text {in }} & \mathrm{N}_{\text {disk }} & \begin{array}{c}\text { Flux } \\ \text { ergs cm }\end{array} & \chi^{2} \mathrm{~s}^{-1} \\ - & - & \mathrm{keV} & - & - \\ \text { 19 June } & 0.75_{-0.02}^{+0.02} & 1.09_{-0.01}^{+0.01} & 75_{-5}^{+5} & 1.310^{-9} & 1.2 \\ \text { 26 June } & 0.79_{-0.02}^{+0.02} & 1.07_{-0.02}^{+0.02} & 56_{-3}^{+4} & 9.010^{-10} & 1.1\end{array}$

Table 1: Spectral parameters of the two ToO pointings: $\mathrm{n}_{h}=$ equivalent hydrogen column, $\mathrm{T}_{i n}=$ inner temperature of the disc, $\mathrm{N}_{d i s k}=$ normalization factor of the diskbb model correlated with the inner radius of the disc.

while IGR J17098-3628 was quite bright. The IGR J17091-3624 upper limit luminosity in $570 \mathrm{~s}$ of observation is of $10^{33} \mathrm{ergs} \mathrm{s}^{-1}$.

Thus second pointing, centered on IGR J17098-3628, was performed in window timing mode in order to avoid pileup (2008-09-19). The third and last one in window timing mode was performed 7 day after (2008-09-26). Figure Ø shows the lightcurve between 2-10 keV of IGR J170983628 of the first pointing of the XRT ToO; the source flux increased with time while in the subsequent pointings the flux was mostly constant.

\subsection{Spectral analysis}

Only the last two pointings were used in this work to extract the IGR J17098-3628 spectra. The analysis showed that the source had still the same spectral shape of the previous observation campaigns in 2005, 2006 and 2007 [1]. The soft spectrum is also confirmed by the lack of any INTEGRAL/IBIS detection above $20 \mathrm{keV}$. The best fit of each pointing is an absorbed disk black body model (diskbb in XSPEC [П]) with a temperature of about $1 \mathrm{keV}$, an internal radius proportional to the last stable orbit and an absorption consistent with the galactic one. The flux slighly varies from $1.3 \times 10^{-9} \mathrm{ergs} \mathrm{cm}^{-2} \mathrm{~s}^{-1}$ (19 June) to $9.1 \times 10^{-10} \mathrm{ergs} \mathrm{cm}^{-2} \mathrm{~s}^{-1}$ (26 June). Figure 3 shows the unfolded spectrum of the 19 June pointing, while in Table 1 the spectral parameters of the two pointings are reported. 


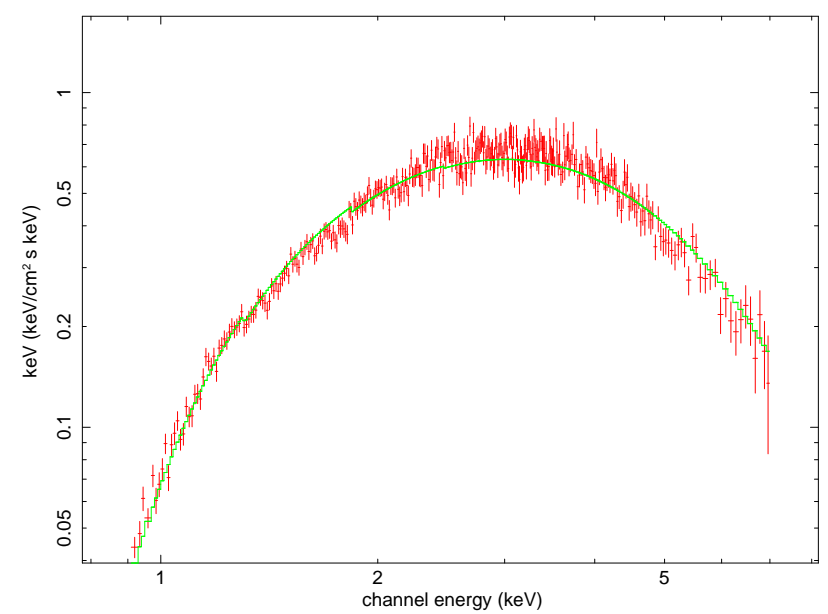

Figure 3: Swift/XRT unfolded spectrum of IGR J17098-3628 during the 2008 June 19 ToO pointing.

\section{Infrared archives data}

IGR J17098-3628 has been previously associated with 2MASS 17094612-3627573 [2], while the XRT refined position of IGR J17091-3624 [3] allowed the association with a new radio counterpart detected at the sub-mJy level in a VLA $8.4 \mathrm{GHz}$ image [1]. The VLA observation was taken 9 days after the first detection of the source by IBIS in 2003. This radio source, not consistent with the one previously proposed by Rupen et al. in 2003, has a typical spectrum of a BHC in low hard states [1]. We used the new radio position to search the source in 2MASS catalogue and in the Spitzer/IRAC survey data and we found a faint infrared source in either catalogues consistent with the radio error box as the two panels of Figure 7 show.

\section{Conclusions}

Our analysis proves that the source spectrum of IGR J17098-3628 shows a soft black body component with an internal temperature of about $\sim 1 \mathrm{keV}$ and an internal radius comparable to the last stable orbit of the accretion disc. As reported by Capitanio et al. 2009 this source spent 3 years in high soft state with a disc black body component substantially identical to the one previously observed at the beginning of the outburst [2]. The only difference is the lack of any high energy emission. In fact the hard component fell below the detection limit of IBIS after about three months from the beginning of the 2005 outburst. Hence the geometry and the temperature of the accretion disc have not shown any significant variation up to now, on the other end the power law emission quenched probably because of the electron temperature of the corona fallen below the disc seed photons temperature making the inverse Compton scattering processes inefficient.

IGR J17091-3624 was not detected during the 2008 Swift ToO being the source probably in quiescent state. Anyway, using the refined radio position previously reported by Capitanio et al 2009, we identified a possible infrared counter part of IGR J17091-3624. Further infrared observations could better identifyed the nature of the source counterpart. 


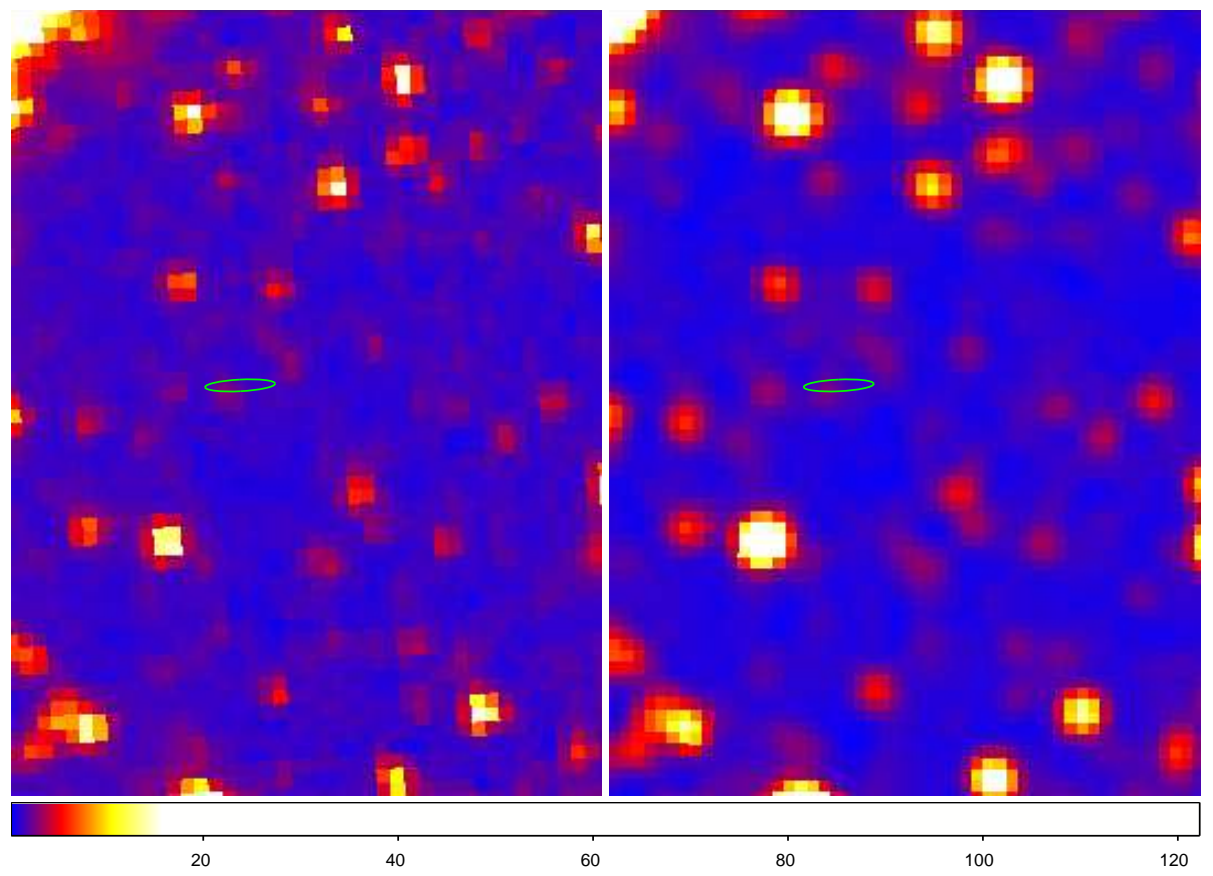

Figure 4: Right panel:Spitzer/IRAC GLIMPSE survey data [3.6 $\mu \mathrm{m}]$ band. The green ellipse is the Radio error box (90\% confidence) of IGR J17091-3624. A faint source is within the error box. Left panel: the same source has been detected in the 2MASS $([1,25 \mu \mathrm{m}])$

\section{References}

[1] Capitanio, F., Giroletti, M., Molina, M. et al. 2009, ApJ, 960, 162

[2] Grebenev, S., A., Molkov, S., V., Revnivtsev, M., G. \& Sunyaev, 2007, ESA SP, 622, 373 (astro-ph: 0709.2313)

[3] Kennea, J., A. \& Capitanio, F. 2007 Atel 1140

[4] Mitsuda, K., Inoue, H., Koyama et al., 1984, PASJ, 36, 741

[5] Rupen, M. P., Mioduszewski, A. J.\& Dhawan, V., 2003 Atel 152. 